\section{Aspergilosis esofágica en una paciente con leucemia mieloide aguda y neutropenia febril}

\author{
Santiago Besa, Eduardo Kattan, Ximena Cid y Juan C. Claro
}

Esophageal aspergillosis in a patient with acute myelogenous leukemia and febrile neutropenia

Aspergillosis usually compromises the respiratory system, but can also affect others. We report a 46 yo female with acute myeloid leukemia, developed febrile neutropenia and dysphagia. Endoscopy revealed esophageal cytomegalovirus-like ulcers, but biopsies showed Aspergillus spp. It's important to consider aspergillosis in the differential diagnosis of esophageal lesions in high-risk patients.

\section{Introducción}

L a aspergilosis invasora (AI), causada por hongos filamentosos del género Aspergillus, se caracteriza por la invasión de vasos sanguíneos, produciendo infartos, necrosis y en consecuencia, diseminación a distintos órganos. Compromete frecuentemente pulmón y senos paranasales. Sin embargo, están descritos también el compromiso dermatológico, oftalmológico, cardíaco y gastrointestinal ${ }^{1}$. Se presenta principalmente en pacientes inmunocomprometidos, con neutropenia secundaria a la quimioterapia por cáncer ${ }^{1}$. Presentamos el caso clínico de una paciente con AI con compromiso esofágico.

\section{Caso clínico}

Paciente de sexo femenino de 46 años, sin antecedentes mórbidos. Consultó por una semana de dolor torácico punzante, fiebre y tos productiva. Además refería un mes de disfagia lógica, baja de peso (siete $\mathrm{kg}$ ) y sudoración nocturna.

Se tomó un hemograma que mostró una hemoglobina $6,2 \mathrm{~g} / \mathrm{dl}$, leucocitos $9.830 / \mathrm{mm}^{3}$, plaquetas $77.200 / \mathrm{mm}^{3}$, PCR $216 \mathrm{mg} / \mathrm{l}$ y una radiografía de tórax demostró una consolidación en el lóbulo superior derecho.

Se hospitalizó para estudio de la bicitopenia, disfagia y síndrome consuntivo. Se diagnosticó una neumonía bacteriana por lo que recibió ceftriaxona por 10 días, con buena respuesta clínica.

Dentro del estudio de la disfagia se realizó una endoscopía digestiva alta (EDA) que mostró tres úlceras esofágicas sugerentes de etiología viral (citomegalovirus) (CMV) (Figura 1). Se tomaron biopsias y se inició tratamiento con ganciclovir por 10 días, con escasa respuesta clínica. El estudio histológico se informó como una erosión mucosa sin signos citopáticos de CMV. La RPC en sangre para CMV también fue negativa.

La bicitopenia fue confirmada con un hemograma con neutropenia de 200 neutrófilos $/ \mathrm{mm}^{3} ; 88 \%$ de blastos y un mielograma que informó una leucemia mieloide aguda (LMA). Una vez finalizados los tratamientos antimicrobianos inició quimioterapia.

Escuela de Medicina, Pontificia Universidad Católica de Chile. Santiago, Chile. (SB, EK, XC, JCC)

Recibido: 13 de octubre de 2013 / Aceptado: 28 de enero de 2014.

Correspondencia a:

Juan C. Claro

jcclarog@uc.cl

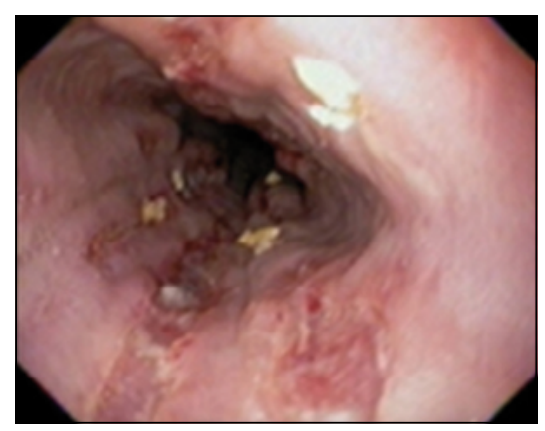

Figura 1. Endoscopía digestiva alta. Se observan tres úlceras en tercio medio esofágico, cubiertas con exudado que se desprende al lavar y dejan fondo de fibrina de hasta $20 \mathrm{~mm}$.

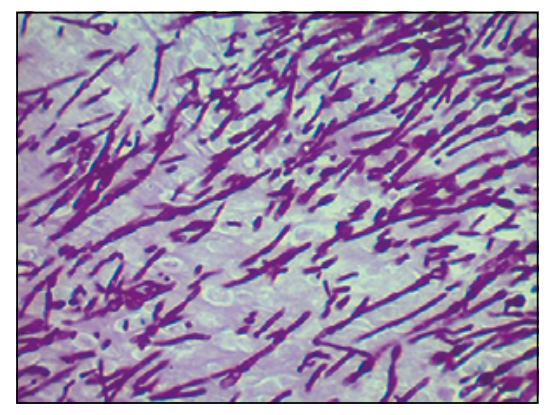

Figura 2. Biopsia de tejido esofágico en microscopía óptica. Tinción de ácido peryódico de Schiff. Se observan hifas septadas ramificadas en $45^{\circ}$, compatibles con Aspergillus spp.

Tres días después de la quimioterapia presentó un episodio de neutropenia febril (NF) con un RAN de 20 neutrófilos $/ \mathrm{mm}^{3}$. Una nueva radiografía de tórax no mostró cambios con respecto a la anterior. Inició tratamiento con imipenem y vancomicina i.v. Por persistencia de la fiebre y la neutropenia, al cuarto día se repitieron los cultivos y se realizó una TAC pulmonar, abdominal y de pelvis. La TAC pulmonar mostró un infiltrado en el lóbulo superior derecho con "signo del halo". Se inició voriconazol sospechando una aspergilosis pulmonar. Los hemocultivos y los galactomananos en sangre resultaron negativos.

Por persistencia de la disfagia, una nueva EDA mostró persistencia de las úlceras esofágicas. El estudio histológico evidenció hifas septadas, ramificadas en $45^{\circ}$, sugerentes de Aspergillus spp (Figura 2).

Luego de dos semanas de tratamiento antifúngico la paciente se encontraba sin fiebre y sin disfagia. Se completaron 18 días de tratamiento antibacteriano y 21 días de voriconazol.

\section{Discusión}

El compromiso gastrointestinal en la AI es infrecuente, presentándose aisladamente en menos de $5 \%{ }^{2}$. Cursa con sintomatología inespecífica (disfagia, hematoquezia, obstrucción intestinal, etc.) secundaria a una ulceración, necrosis o abscesos. Generalmente se asocia al compromiso concomitante de otros órganos, principalmente pulmón. Una revisión en pacientes hematológicos mostró que dentro del compromiso gastrointestinal, el compromiso de colon e intestino delgado fueron los más frecuentes (36 y $41 \%$, respectivamente) y sólo $9 \%$ presentó compromiso esofágico ${ }^{3}$.

La AI gastrointestinal es difícil de objetivar. En dicha revisión, 57\% de las AI con compromiso gastrointestinal presentó al menos una medición de galactomanano elevada ${ }^{3}$. El cepillado citológico complementaría el diagnóstico por biopsia, pero aún no ha sido validado ${ }^{4}$. 
Existen otros casos reportados de aspergilosis esofágica ${ }^{5-7}$. Todos coinciden en la presentación con síntomas inespecíficos (disfagia, pirosis) en pacientes profundamente inmunocomprometidos, la importancia diagnóstica con estudio histológico y cultivo, y la buena respuesta a tratamiento con antifúngicos (voriconazol, caspofungina).

Nuestro caso clínico presenta una manifestación poco frecuente de AI. El compromiso aislado del esófago constituye una rareza. Por esta razón, la sospecha debe enfocarse en pacientes con síntomas esofágicos con otro foco probable o confirmado de AI.

En pacientes con factores de riesgo de AI y con presencia de úlceras esofágicas debe considerarse la aspergilosis esofágica como diagnóstico diferencial, en especial en aquellos en que fracasa el tratamiento de las etiologías más probables como CMV.

\section{Referencias bibliográficas}

1.- Pagano L, Caira M, Candoni A, Offidani M, Martino B, Specchia G, et al. Invasive aspergillosis in patients with acute myeloid leukemia: a SEIFEM-2008 registry study. Haematologica 2010; 95: 644-50.
2.- Eggimann P, Chevrolet J C, Starobinski M, Majno P, Totsch M, Chapuis B, et al. Primary invasive aspergillosis of the digestive tract: report of two cases and review of the literature. Infection 2006; 34: 333-8.

3.- Kazan E, Maertens J, Herbrecht R, Weisser M, Gachot B, Vekhoff A, et al. A retrospective series of gut aspergillosis in haematology patients. Clin Microbiol Infect 2011; 17: 588-94.

4.- Bergman S, Geisinger K R. Esophageal aspergillosis in cytologic brushings: report of two cases associated with acute myelogenous leukemia. Diagn Cythopathol 2004; 30: 347-9.

5.- Chionh F, Herbert K E, Seymour J F, Prince H M, Wolf M, Zimet A, et al. Ante-mortem diagnosis of localized invasive esophageal aspergillosis in a patient with acute myeloid leukemia. Leuk Lymphoma 2005; 46: 603-5.

6.- Akyol Erikci A, Ozyurt M, Terekeci H, Ozturk A, Karabudak O, Oncu K. Esophageal aspergillosis in a case of acute lymphoblastic leukaemia successfully treated with caspofungin alone due to liposomal amphotericin B induced severe hepatotoxicity. Mycoses 2009; 52: 84-6.

7.- $\quad$ Alioglu B, Avci Z, Canan O, Ozcay F, Demirhan B, Ozbek N. Invasive esophageal aspergillosis associated with acute myelogenous leukemia: successful therapy with combination caspofungin and liposomal amphotericin B. Pediatr Hematol Oncol 2007; 24: 63-8. 